\title{
Some results on a black hole with a global monopole in Poincaré gravity
}

\author{
Valdir B. Bezerra ${ }^{1, a}$, Cristine N. Ferreira ${ }^{2, b}$, Elton P. J. Alvarenga ${ }^{2, c}$ \\ ${ }^{1}$ Departamento de Física, Universidade Federal da Paraíba, Caixa Postal 5008, João Pessoa, PB 58059-970, Brazil \\ ${ }^{2}$ Núcleo de Estudos em Física, Instituto Federal de Educação, Ciência e Tecnologia Fluminense, Rua Dr Siqueira 273, Campos dos Goytacazes, \\ RJ 28030-130, Brazil
}

Received: 3 April 2018 / Accepted: 23 May 2018 / Published online: 4 June 2018

(C) The Author(s) 2018

\begin{abstract}
The aim of this work is to study the thermodynamics and spin current of a system corresponding to a black hole containing a global monopole in the context of Poincaré gravity theory which is an extension of general relativity, in the sense that the intrinsic angular momentum of matter is also a source of gravitational interaction. Thus, in this work we find the solution corresponding to the spacetime under consideration by taking into account that the action which describes this system contains terms corresponding to the curvature and torsion. The metric obtained is a function of mass, solid angle deficit and the coupling constants of the quadratic terms of the curvature and torsion. In this model, the stability of the system is studied through the analysis of the Hawking temperature and the specific heat. In this context it was also studied the critical temperatures of the system considering positive or negative cosmological constant. In the vicinity of the black hole with a global monopole, where there is a logarithmic correction due to the relationship between the torsion and curvature fields, some analysis were done. We also study the AdS/dS limit where the black hole is analyzed from the topological point of view. Although the effect of spin current density at low energies is negligible, in the vicinity of strong gravitational fields it can generate an appreciable effect due to spin gravity coupling.
\end{abstract}

\section{Introduction}

One of the most intriguing phenomena that can be observed in the universe concerns to the fact that it experiences an accelerated expansion, a phenomenon confirmed recently from the observations of supernova explosions. Due to these observa-

\footnotetext{
a e-mail: valdir@fisica.ufpb.br

b e-mail: crisnfer@msn.com

c e-mail: elton.apj@gmail.com
}

tions with supernovae it has been concluded that the universe nowadays is expanding rapidly. One idea to explain this acceleration is that the universe is dominated by dark energy that seems to be more abundant than the usual energy $[1,2]$.

A similar scenario with respect to the occurrence of acceleration also appears in the primordial stages of the universe. At that period, it experienced a great acceleration called inflation $[3,4]$, but in those times the universe was very hot and dense and only at the end of this inflationary period, the structures began to be formed $[5,6]$.

Although the general relativity is undoubtedly the most promising theory to explain most of the events occurring in the universe, many questions still seem inexplicable, as for example the accelerated expansion. Another theoretical point is the fact that there is no a theory of quantum gravity based on general relativity. Thus, some theories were proposed to unify the formalism of the quantum field theory with gravitational formalism, as for example, the EinsteinCartan theory. This theory, extends the theory of general relativity by including a connection that takes into account its antisymmetric part. It was in this period that the concept of torsion in general relativity was born. The great achievement of Poincar's gravity gauge theory is that besides the source of gravity corresponding to energy-momentum, the intrinsic angular momentum or the spin of matter is also a source of gravitational interactions. In this way, the resulting spacetime geometry is endowed with curvature and torsion [7]. Although the effects of spin are negligible at low energies, certainly, it was very important in the primordial universe when spin density was very high [8].

We also know that local or global change of the universe implies in phase transitions that can originate from breaks of symmetry, in analogy to what occurs in thermodynamic systems. One of the most important laboratories for physics 
is the universe and one of the most intriguing objects investigated are the black holes [9].

It can carry mass, charge, angular momentum and can be described by thermodynamic laws [10-13]. The implications of the studies related to this object can undoubtedly help us to understand a greater number of issues. However, so far black hole physics has been dependent on the models, the observations can provide data that discards certain models and reinforces others. For this reason the study of black holes in various contexts is of paramount importance [14-16]. In special, we will consider a black hole with a topological defect, namely, global monopole. This topological has been formed at phase transitions in the very early universe. The gravitational field of a global monopole possesses a deficit solid angle which is proportional to the energy scale of symmetry breaking [17].

Today it is believed that the universe is de-Sitter (dS) with a small positive cosmological constant. Many investigations have appeared in the literature considering the cosmological constant to be the negative pressure necessary to make the universe to expand in accelerated way [18]. Taking into account the presence of a cosmological constant, some other studies consider the black hole in Anti- de-Sitter (AdS) spaces. Despite that for this space-time, the cosmological constant is negative, it can be included from the point of view of the relation between an alien world with a border according to which it could represent our particle physics [19]. This mechanism became known as AdS/CFT correspondence [20-22]. From the point of view of physics of a black hole physics with AdS spacetime, we can relate the entropy with the area of the black hole surface [13]. In this formalism, the Hawking-Page phase transitions can be studied [23]. The black hole in an AdS spacetime give us the correct relation between temperature and the specific heat which is important to understand the black hole thermodynamics.

In this work a different context will be proposed to study black hole thermodynamics, in such a way that it will taken into account that the space time besides curvature has also torsion. The theory is called Poincaré, gravity because the connections that make up the Riemann tensor are considered as gauge fields and contain information arising from the metric as well as from the torsion. For consistency of the equations, in order to have a torsion that can propagate, we will use the formalism where two quadratic terms are introduced, one for the Riemann tensor and another pair for the torsion tensor $[24,25]$. One of the advantages of working with this model is the appearance of spin chains. These spin currents come from the conception that the derivative of the action in relation to torsion can give us spin chains. Thus, it should be important to study the coherence of this system [26]. Another advantage of this model is to better understand the de-Sitter space, through a correct relation between tem- perature and specific heat, analogous to what happens in the Anti-de-Sitter case. In fact, in this model, one can consider de-Sitter and Anti-Sitter as experiencing a Hawking-Page type phase transitions.

This paper is organized as follows. In Sect. 2, we present more global aspects Poincar gravity. In Sect. 3, we perform an analysis of thermodynamics of the system under consideration. In Sect. 4, we discuss the spin current. Finally, in Sect. 5, we present the conclusions.

\section{Description of the Poincaré-gravity framework}

In this section we present the basic ingredients of the Poincaré gravity, including two quadratic terms, one of these is the torsion and the other is the curvature. The full action that contains both gauge fields are given by

$S=S_{g}+S_{\epsilon}$,

where the Poincare gravity action is written as

$S_{g}=\frac{1}{\kappa} \int \sqrt{-g} d^{4} x\left[R-\frac{\alpha}{4} R_{\mu \nu}^{a b} R_{a b}^{\mu \nu}-\frac{\beta}{4} \mathcal{Q}_{\mu \nu}^{a} \mathcal{Q}_{a}^{\mu \nu}\right]$,

with $\alpha$ and $\beta$ being constants and $\mu, v=0,1,2,3$ and $a$, $b=0,1,2,3$ being spacetime indices and tetrad indices, respectively. The quantities $R_{\mu \nu}^{a b} e_{b}^{\mu} e_{a}^{\nu}$ and $g_{\mu \nu}=e_{\mu}^{a} e_{\nu}^{b} \eta_{a b}$ are, respectively, the curvature scalar and the metric associated with the gravitational field. The Riemann and the torsion tensor field are given by

$$
\begin{aligned}
R_{\mu \nu}^{a b} & =\partial_{[\mu} \Gamma_{\nu]}^{a b}+\Gamma_{\mu c}^{a} \Gamma_{\nu}^{c}{ }^{b}, \\
\mathcal{Q}^{a}{ }_{\mu \nu} & =\partial_{[\mu} e_{\nu]}^{a}+\Gamma_{\mu b}^{a} e_{\nu}^{b} .
\end{aligned}
$$

In the approach considered, these fields are gauge field strengths related with the connection $\Gamma_{v}{ }^{a b}$ and the vierbein $e_{\mu}^{a}$, as explicitly shown in Eqs. (3) and (4).

The general form of the time independent metric with spherical symmetry in $(3+1)$ dimensions is given by

$d s^{2}=-e^{2 \mu} d t^{2}+e^{2 v} d r^{2}+r^{2}\left(d \theta^{2}+\sin \theta^{2} d \phi^{2}\right)$,

where $\mu=\mu(r)$ and $\nu=v(r)$, which means that these functions depend only on the radial coordinate.

In this case, the non-vanishing independent components of torsion tensor are given by

$\mathcal{Q}_{01}^{0}=f(r), \quad \mathcal{Q}_{20}^{2}=\mathcal{Q}_{30}^{3}=k(r)$,

$\mathcal{Q}^{1}{ }_{10}=h(r), \quad \mathcal{Q}^{2}{ }_{21}=\mathcal{Q}_{31}^{3}=-g(r)$,

where $f(r), g(r) h(r)$ and $k(r)$ are function to be determined and the Riemann-Cartan affine connection, $\tilde{\Gamma}$, is given by $2 \tilde{\Gamma}_{i}{ }^{k}{ }_{j}=-\Gamma_{i}{ }^{k}{ }_{j}+\mathcal{Q}_{i}{ }^{k}{ }_{j}$, where $\Gamma_{i}{ }^{k}{ }_{j}$ corresponds to the Riemann part and $\mathcal{Q}_{i}{ }^{k}{ }_{j}$ to the contribution of the torsion. 
The nonvanishing independent curvature components $R^{i}{ }_{j k l}$ are given by

$$
\begin{aligned}
& R_{110}^{0}=\left(\mu^{\prime} e^{\mu-v}+e^{\mu} f\right)^{\prime} e^{-\mu-v} \equiv A(r), \\
& R_{323}^{2}=\frac{1}{r^{2}}+k^{2}-\left(\frac{1}{r} e^{-v}-g\right)^{2} \equiv B(r), \\
& R_{202}^{0}=R_{303}^{0}=-\left(f+\mu^{\prime} e^{-v}\right)\left(\frac{e^{-v}}{r}-g\right) \equiv C(r), \\
& R_{212}^{1}=R_{313}^{1}=\frac{1}{r} e^{-v}\left(r g-e^{-\mu}\right)^{\prime}+h k \equiv D(r), \\
& R_{202}^{1}=R_{303}^{1}=\left(v^{\prime} e^{-v}+f\right) k \equiv E(r), \\
& R_{212}^{0}=R_{313}^{0}=(r k)^{\prime} \frac{e^{-v}}{r}-h\left(\frac{1}{r} e^{-v}-g\right) \equiv-F(r) .
\end{aligned}
$$

In order to write equations in a simplest way, we are identifying the components of the Riemann tensor with the functions $\mathrm{A}(\mathrm{r}), \mathrm{B}(\mathrm{r}), \ldots$.

The equations of the motion written in terms of the vierbein, are given by

$G_{i j}=R_{i j}-\frac{1}{2} \eta_{i j} R=-\kappa T_{i j}-\alpha \mathcal{T}_{i j}-\beta \mathcal{Q}_{i j}$,

where

$$
\begin{aligned}
\mathcal{T}_{i j} & =-\frac{1}{2} R_{i l m}{ }^{n} R_{j}{ }^{l m}{ }_{n}+\frac{1}{8} \eta_{i j} R_{i l m}{ }^{n} R^{i l m}, \\
\mathcal{Q}_{i j} & =-\frac{1}{2} \mathcal{Q}_{i m}{ }^{n} \mathcal{Q}_{j}{ }^{m}{ }_{n}+\frac{1}{8} \eta_{i j} \mathcal{Q}_{i m}{ }^{n} \mathcal{Q}^{i m}{ }_{n} .
\end{aligned}
$$

The action of matter corresponding to the defect is $S_{\epsilon}$. An interesting type of defect that can be related with the black hole is the global monopole with a $O(3)$ symmetry broken. The action associated to the matter field coupled with gravity that represents the global monopole is

$S_{\epsilon}=\frac{1}{2} \int d^{4} x \sqrt{-g}\left[\left(\partial_{\mu} \phi^{a}\right)\left(\partial^{\mu} \phi^{a}\right)-\frac{1}{2} \lambda\left(\phi^{a} \phi^{a}-\eta^{2}\right)^{2}\right]$,

where $\lambda$ is the self-interaction constant, $\eta$ is the scale of a gauge-symmetry breaking and the triplet field that will result in a monopole configuration can be described by $\phi^{a}=\eta \frac{\varphi(r)}{r^{2}} x^{a}$, with $a=1,2,3$ and $x^{a} x^{a}=r^{2}$. The function $\varphi(r)$ is dimensionless and constrained by the condition $\varphi(0)=0$ and $\varphi(r>\eta) \sim 1$.

The energy momentum tensor is given by

$T_{\mu}^{i}=\frac{1}{2 \sqrt{-g}} \frac{\delta S_{\epsilon}}{\delta e_{i}^{\mu}}$.

Considering that the monopole is formed in the black hole background, we have that the energy momentum tensor is given by
$T_{00}=-T_{11} \sim \frac{\eta^{2}}{r^{2}}=\frac{\epsilon}{\kappa r^{2}}$,

$T_{22}=T_{33}=0$.

We are considering that the Einstein tensor components are function of the metric and torsion. The contribution of the torsion can be understood if we analyze the Riemann tensor given by Eq. (4). The connection $\Gamma_{v}{ }^{a b}$ has two parts: one related to the metric and the other to the torsion. The non-vanishing components of the Einstein tensor are $G_{00}$, $G_{11}, G_{22}=G_{33}$, in which case there are off-diagonal terms due to the presence of the torsion. This fact implies that in energy-momentum tensor has also off-diagonal componentes, namely, $G_{01}$ and $G_{10}$.

If we analyze these components we can observe that when the torsion components are zero, then, the off-diagonal components of the Einstein tensor are zero, and as a consequence, the components of the Einstein tensor become the ones corresponding to the usual global monopole in a black hole background. The non-vanishing quadratic terms of the energymomentum tensor associate to the curvature are $\mathcal{T}_{00}, \mathcal{T}_{11}$, $\mathcal{T}_{22}$.

With torsion fields the off-diagonal components of the energy-momentum tensor are not zero, even when the metric is diagonal. This fact produces important consequences on the geodesics of the test particles in the spacetime generated by the system formed by the black hole and the defect, specially when the torsion is taken into account. The offdiagonal components of the energy-momentum tensor are $\mathcal{T}_{10}=\mathcal{T}_{01}$ when $k=h=0$ and the off-diagonal components of the energy-momentum tensor become zero.

The Einstein equations in weak field approximation are given by

$$
\begin{aligned}
G_{00}= & -2 D(r)-B(r)=-\alpha(2 x y+u v) \\
& -\frac{1}{2} \beta\left[f^{2}-h^{2}-2\left(k^{2}+g^{2}\right)\right]-\frac{\epsilon}{r^{2}}, \\
G_{11}= & B(r)-2 C(r)=-\alpha(2 x y-u v) \\
& -\beta\left(k^{2}+g^{2}\right)+\frac{\epsilon}{r^{2}},
\end{aligned}
$$

$G_{22}=G_{33}=A-y=-\alpha u v+\frac{1}{2} \beta\left(f^{2}-h^{2}\right)$,

$G_{01}=G_{10}=4 \alpha E y-2 \beta k$,

with $E(r)=-F(r), x=C(r)+D(r), y=C(r)-D(r)$, $u=A(r)+B(r)$ and $v=A(r)-B(r)$, where $\mathrm{A}(\mathrm{r}), \mathrm{B}(\mathrm{r})$, $\mathrm{C}(\mathrm{r}), \mathrm{D}(\mathrm{r}), \mathrm{E}(\mathrm{r}), \mathrm{F}(\mathrm{r})$ are given by Eqs. (7)-(12).

In this work we assume that the functions which appear in Eq. (6) are such that $g=k, h=-f$, and the metric parameters, $\mu$ obey the usual relation $v \mu=-v$. The condition $y=-\frac{1}{4 \alpha}$ in the Eq. (22) gives us

$\mu^{\prime} e^{2 \mu}-h e^{\mu}=-\frac{2}{3} \beta k e^{\mu}$, 
and using the relation $E=-F$, we have

$-\frac{k}{r}-k^{\prime}+\frac{h}{r}=\mu^{\prime} k$

Using these assumptions and results together with Einstein's equations given by Eqs. (19) and (20), we get

$u=4 y+\frac{\epsilon}{r^{2}}=0$,

which supplies the following relation

$\left(\mu^{\prime} e^{2 \mu}\right)^{\prime}+2\left(f e^{\mu}\right)^{\prime}+\frac{4 k e^{\mu}}{r}-2 \frac{e^{2 \mu}}{r^{2}}+2 \frac{(1-\epsilon)}{r^{2}}=-\frac{1}{2 \alpha}$

The solution of these equations are

$$
\begin{aligned}
e^{2 \mu} & =1-\epsilon-\frac{2 G M}{r}+\frac{r^{2}}{L^{2}}+N r^{2} \ln r, \\
k & =\left[C_{1} r+\frac{C_{2}}{r^{2}}+\frac{r}{2 \alpha \beta} \ln r\right] e^{-\mu}, \\
h & =\left[\left(2 C_{1}+\frac{1}{2 \alpha \beta}\right) r+\frac{C_{2}}{r^{2}}+\frac{r \ln r}{\alpha \beta}\right] e^{-\mu},
\end{aligned}
$$

where

$2 G M=2\left(\frac{2}{3} \beta+1\right) C_{2}, \quad N=\left(\frac{1}{\alpha \beta}-\frac{1}{3 \alpha}\right)$,

$\pm \frac{1}{L^{2}}=\left[6\left(1-\frac{\beta}{3}\right) C_{1}+\frac{1}{2 \alpha}\right]$,

with $C_{1}$ and $C_{2}$ being integration constants. For simplicity, we will work with the metric in the form

$d s^{2}=-B(r) d t^{2}+B(r)^{-1} d r^{2}+r^{2}\left(d \theta^{2}+\sin \theta^{2} d \phi^{2}\right)$,

where

$B(r)=1-\epsilon-\frac{2 G M}{r}+\Lambda \frac{r^{2}}{3}+N r^{2} \ln r$.

$G M$ is the energy of the system, $\Lambda= \pm \frac{3}{L^{2}}$ is the cosmological constant and the term with metric constant parameter $\mathrm{N}$ is the new contribution arising from the torsion. This metric represents the spacetime generated by the system we are considering, which is formed by a black hole with a global monopole in a scenario in which the torsion field is present.

\section{Thermodynamics analysis}

In this section, we analyze the behavior of the energy of the system corresponding the source we are considering, formed by a black hole and a global monopole, as a function of those parameters presented in the last section. The dependence of the energy, GM, as a function of the horizon $r_{H}$, is given by
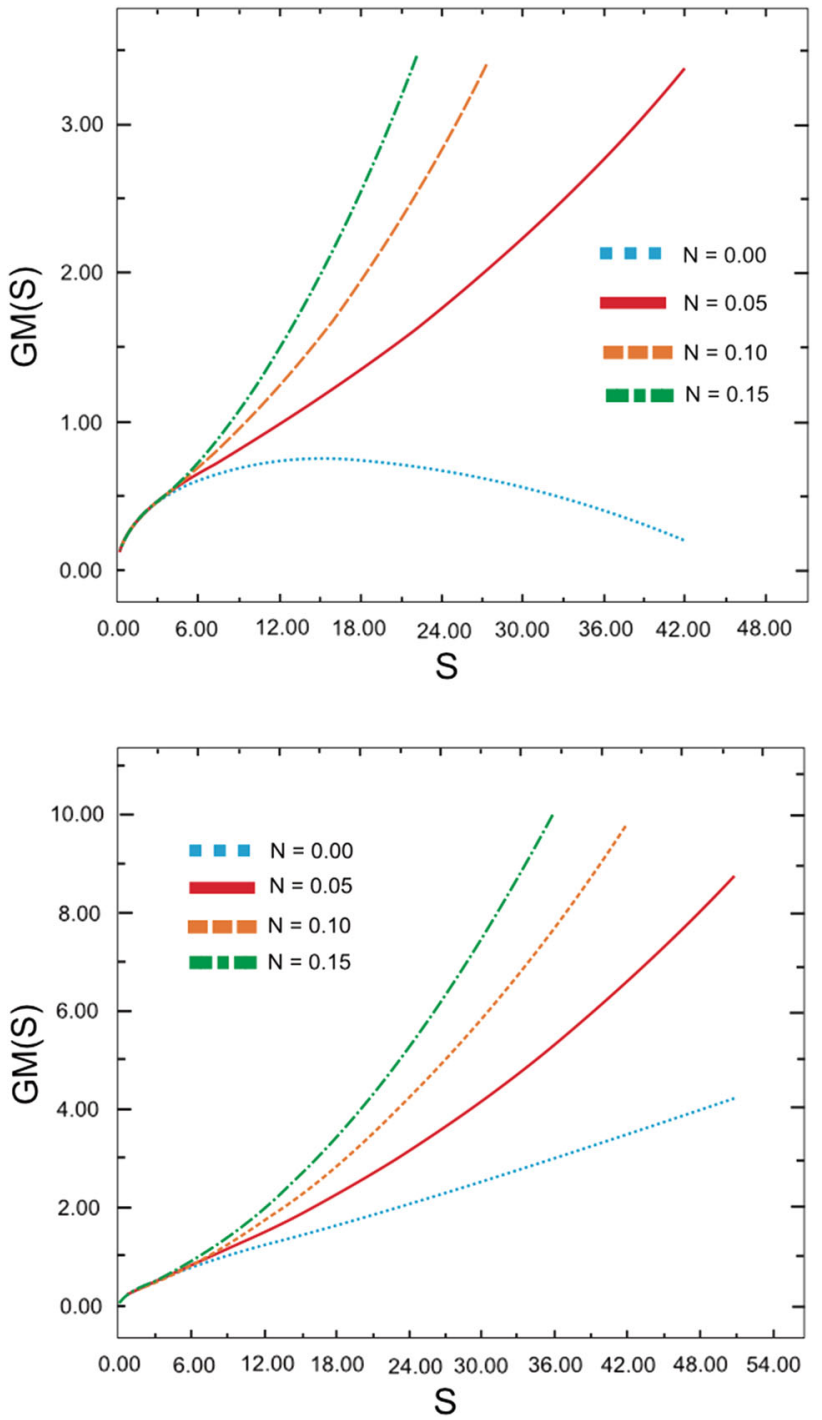

Fig. 1 Graphs for $G M(S) \times S$. In the upper panel, $\Lambda>0$ is taken into account, while in the lower panel, $\Lambda<0$, with $\epsilon=0.5$

$G M=\frac{1}{2} r_{H}\left[1-\epsilon \mp \frac{r_{H}^{2}}{L^{2}}+N r_{H}^{2} \ln r_{H}\right]$

where the minus sign in the third term represents the case with positive cosmological constant, which becomes asymptotically de Sitter (dS) when $r \rightarrow 1$ or $\beta=3$. In the case where this sign is positive, the cosmological constant is negative, and thus when $r \rightarrow 1$ or $\beta=3$, we have the Anti-de-Sitter case.

The behavior of GM, for different N's, as a function of the entropy is shown in Fig. 1, where the entropy is given by $S=\pi r_{H}^{2}$.

The torsion is codified by the parameters $\mathrm{N}$ and $\beta$. When $\mathrm{N}=0$, the metric is, locally $\mathrm{dS}$ (up panel) and AdS (down panel), both represented by the dotted curve. In the upper panel, we can verify that the curve grows with the entropy up 
to a certain value and then decreases. This behavior is very different from the lower panel, in which case the entropy always grows. To analyze the stability of the black hole we need to study the behavior of temperature, specific heat and free energy, which can be done simply by verifying how these magnitudes evolve with the radius of the horizon.

It can be seen that the tangent lines to these curves give us the temperature that satisfies the second law of thermodynamics. From now on we can calculate the Hawking temperature, $T_{H}$, of the system by taking the derivative of the energy with respect to the entropy of the black hole. From the second law of thermodynamics we have that the Hawking temperature is obtained from $d G M=T_{H} d S$, and then we find that

$$
\begin{aligned}
T_{H} & =\left(\frac{d S}{d r_{H}}\right)^{-1} \frac{d G M}{d r_{H}} \\
& =\frac{r_{H}}{4 \pi}\left[\frac{1-\epsilon}{r_{H}^{2}} \mp \frac{3}{L^{2}}+N+3 N \ln \left(r_{H}\right)\right] .
\end{aligned}
$$

In the case where the cosmological constant is negative we still have the possibility of obtaining a black hole either for $N=0$ or with $N \neq 0$. However, for the positive cosmological constant we have the possibility of the existence of stable black hole only when $N \neq 0$. This is the most important feature of this model. We will study in the next sections, in details, both cases, namely, for negative and positive cosmological constants.

In Fig. 2, the behavior of the temperature with the radius of the horizon is showed, when the cosmological constant is positive $\Lambda=\frac{3}{L^{2}}$. In the panel on the upper part two black holes appear when torsion is taken into account. The Hawking temperature decreases as the horizon radius increases, until reaching a minimum value, and than, starts to increase with the radius of the horizon, for $\epsilon=0$ and $\epsilon=0.5$. In the cases where $\epsilon=1.0$ and $\epsilon=1.5$, the Hawking temperature increases with the radius of the horizon. When the black hole is in the presence of a global monopole there appears the phase transition for $0 \leq \epsilon \leq 1.5$. It can be visualized that the behavior of the temperature as a function of the horizon is sensible to the variation of the parameter of the deficit solid angle associated with of the global monopole.

Analyzing this behavior we find that the deficit solid angle has to be less than one. At these limits the temperature decreases to a minimum, showing in this analysis the compatibility of the system. When $\epsilon \geq 1$, the Hawking temperature is negative, which is incompatible with any thermodynamic system. In the lower panel one has the graph of the temperature as a function of the radius of the horizon for the case without torsion, $N=0$. It shows that for small values of the deficit solid angle, the Hawking temperature is positive in a certain region and decreases as the horizon radius $r_{H}$
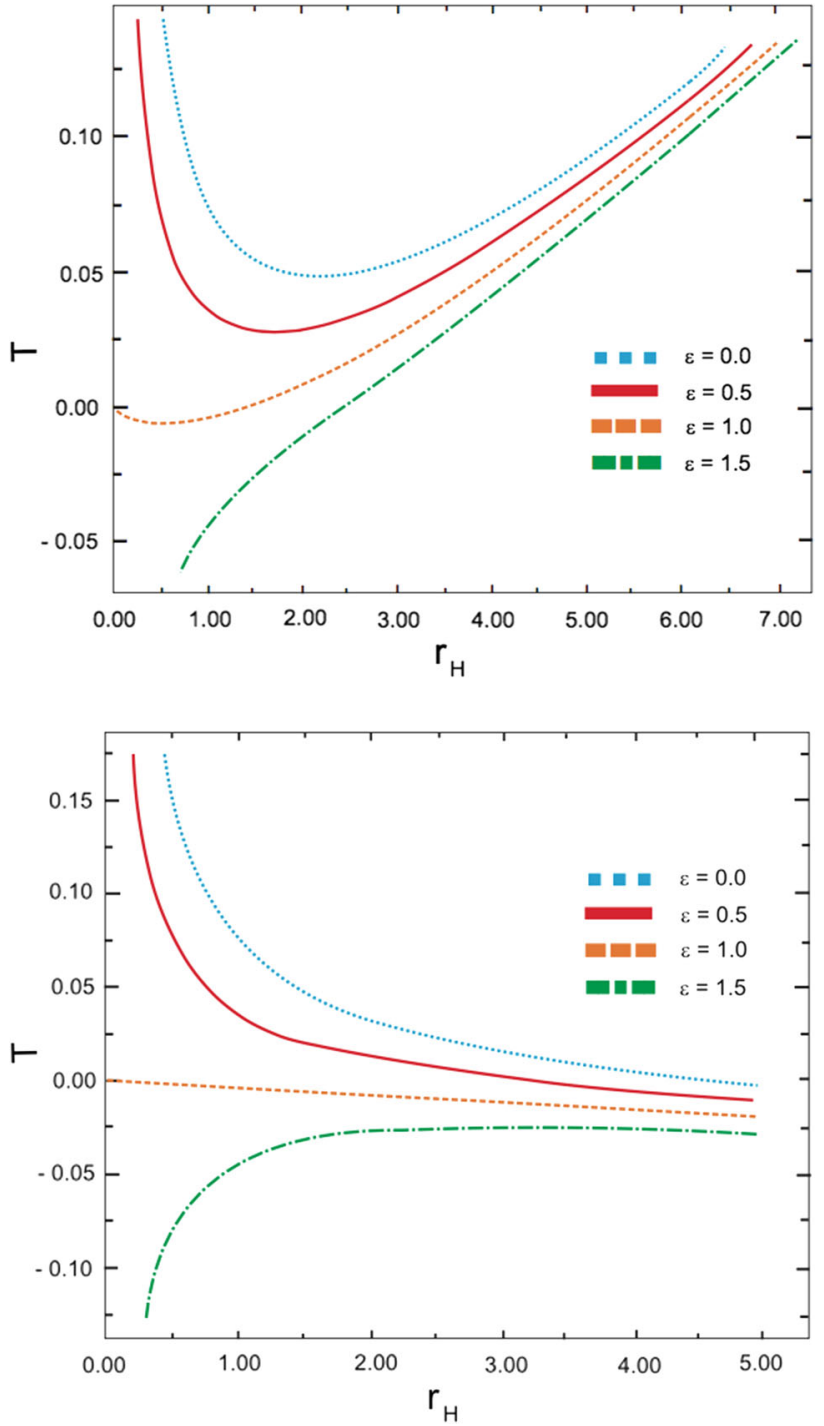

Fig. 2 Graphs for $T \times r_{H}$. In the upper panel, $N=0.5$ and in the lower panel, $N=0$

increases. However, it does not present a minimum value, and thus the black hole is not stable.

Initially we will analyze the critical points of the system, by calculating the minimum of the Hawking temperature, namely, by solving the equation $\left[\frac{\partial T_{H}}{\partial r_{H}}\right]_{r_{H_{\min }}}=0$. To do this, let us use the following relations

$T_{H_{\min }}=\frac{1-\epsilon+\frac{3}{2} N r_{H_{m i n}}^{2},}{2 \pi r_{H_{\min }}}$,

$r_{H_{\text {min }}}=e^{-\frac{1}{6} \frac{-3 N \text { Lambert } W\left(\frac{2}{3}(1-\epsilon) e^{\frac{2}{3} \frac{\mp \Lambda+4 N}{N}}\right) \mp 2 \Lambda+8 N}{N}}$,

where the minimum temperature is such that $T_{H_{\min }}=$ $T_{H}\left(r_{H_{\text {min }}}\right)$ and indicates the existence of a thermal phase transitions between the black holes with the same temperature. 
The interesting effects occur when $N \neq 0$ which is given by $\ln \left(r_{H}\right)$. For temperatures $T<T_{\min }$, there are no black holes, but only radiation. For $T>T_{\min }$ there are multiply black holes, whose horizon, $r_{H}$, associated to them, can be determined by Eq. (34), which gives us the following result

$\left[N-\frac{3}{L^{2}}+3 N \ln \left(r_{H}\right)\right] r_{H}^{2}-4 \pi T_{H} r_{H}+1-\epsilon=0$.

The general expression for the temperature is given by Eq. (34) and the expression for the heat capacity by

$C=\left(\frac{d G M}{d T_{H}}\right)=\left[\frac{d G M}{d r_{H}} \cdot\left(\frac{d T}{d r_{H}}\right)^{-1}\right]$,

where

$\frac{d(G M)}{d r_{H}}=\frac{1}{2}\left[1-\epsilon+\left(N \mp \frac{3}{L^{2}}+3 N \ln \left(r_{H}\right)\right) r_{H}^{2}\right]$,
$\frac{d T}{d r_{H}}=\frac{1}{4 \pi r_{H}^{2}}\left[\epsilon-1+\left(4 N \mp \frac{3}{L^{2}}+3 N \ln \left(r_{H}\right)\right) r_{H}^{2}\right]$.

This result was obtained using the second law of thermodynamics $\mathrm{d}(\mathrm{GM})=\mathrm{TdS}$. In the general case, the positive sign corresponds to the positive cosmological constant, while the negative sign corresponds to the negative cosmological constant. In this scenario, we find that

$C=2 \pi r_{H}^{2}\left[\frac{1-\epsilon+\left(N \mp \frac{3}{L^{2}}+3 N \ln \left(r_{H}\right)\right) r_{H}^{2}}{\epsilon-1+\left(4 N \mp \frac{3}{L^{2}}+3 N \ln \left(r_{H}\right)\right) r_{H}^{2}}\right]$.

Figure 3 shows the heat capacity for different values of $\epsilon=\kappa \eta^{2}$. It can be seen that for $0 \leq \kappa \eta^{2}<1$ the black hole is stable because the condition $T>0$ and $C>0$, are satisfied.

Note that we have $T>T_{\min }$, when $r_{H}>r_{H}^{\min }$, and thus the temperature and the heat capacity are positive and we say that the black hole is stable. In the case where $r_{H}<$ $r_{H_{\min }}$ there is a region where the temperature is positive and the heat capacity is also positive, but in this case the black hole experiences a transition for the equilibrium point. In this region there is a small black hole. The region with the negative heat capacity is the forbidden region.

Then we have an interesting black hole with positive cosmological constant when the torsion is present which is analogue to the AdS black hole. The stable black hole has the temperature increasing with the horizon radius $r_{H}$. This behavior is interesting and means that when the black hole gains energy, as the radius of the horizon $r_{H}$ increases, the temperature also increases.

Another point that can be analyzed, in the graphs of Fig. 3, is the behavior of the curves of heat capacity for different
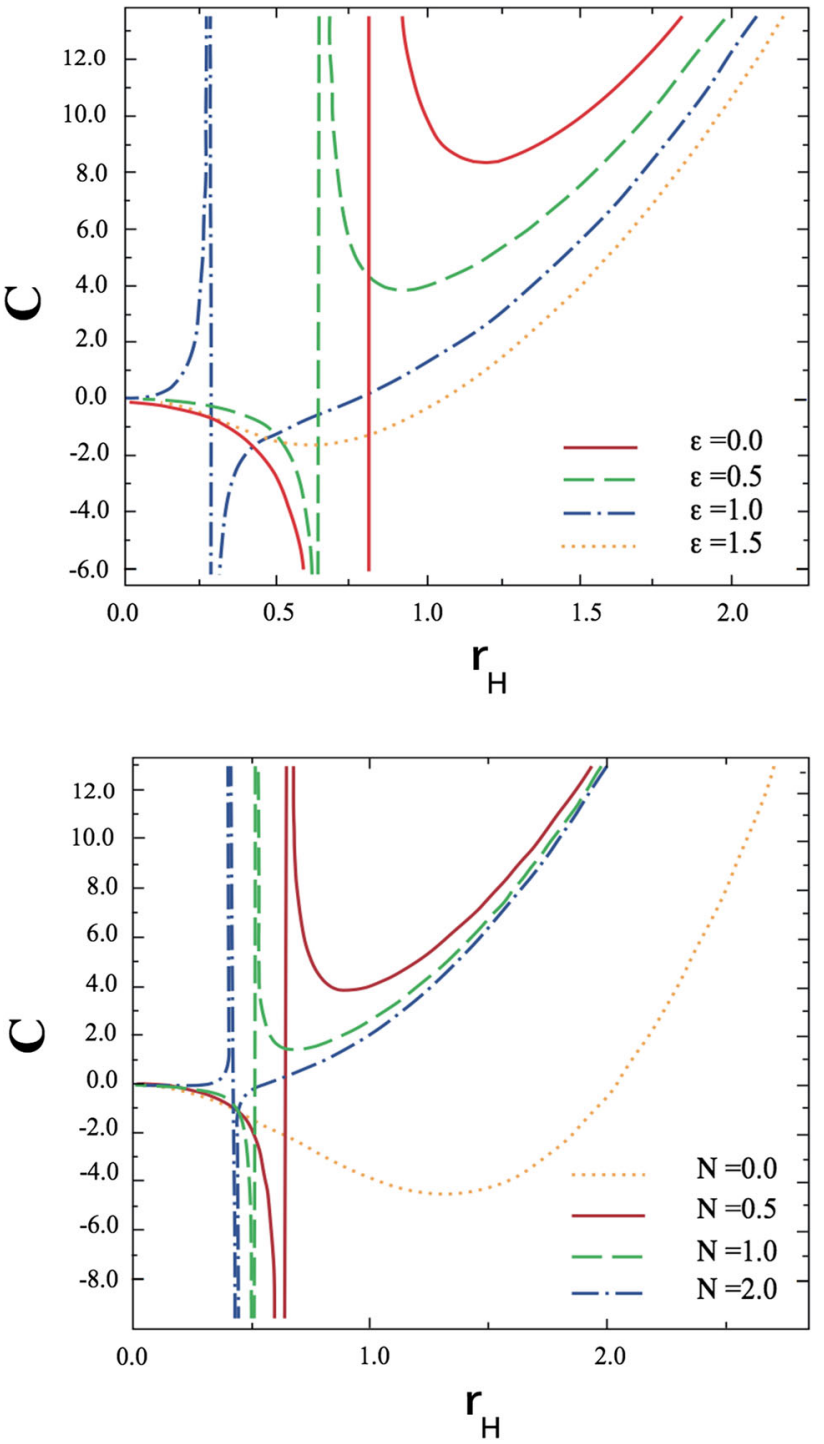

Fig. 3 The Graphs represent $C \times r_{H}$ considering the positive cosmological constant. In the upper panel $N=0.5$ and in the lower panel shows the heat capacity for $\epsilon=0.5$

values of the deficit solid angle. Comparing the heat capacity of the upper and lower panels we can see that it is possible to obtain the same behavior by varying the values of the deficit solid angle or the contribution due to the torsion.

We can verify that the curve $\mathrm{N}=0$ behaves similarly to $\epsilon=1.5$, while $\mathrm{N}=2$ is similar to $\epsilon=1$ and $\mathrm{N}=0.5$ and 1 are similar to $\epsilon=0$ and 0.5 , respectively.

\subsection{The Hawking-Page phase transitions}

Another important quantity to analyze the stability of the black hole is the Hawking-Page phase transition. This transition, at a constant volume, occurs at a point where the Helmholtz free energy, given by $F=G M-T S$, is exactly equal to zero. 


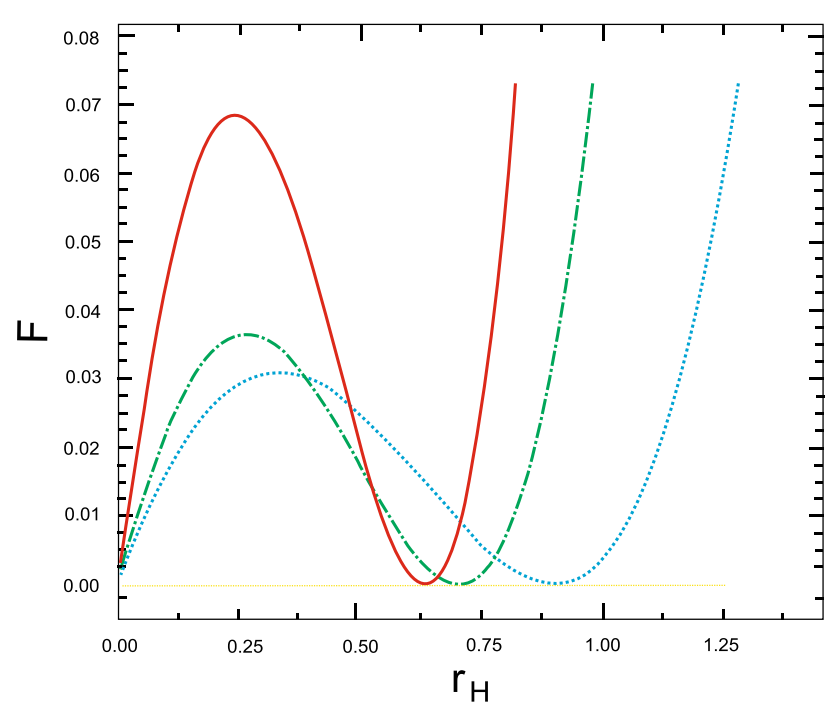

Fig. 4 Graphs of $F \times r_{H}$ in the case of the positive cosmological constant with $T_{H P}=0.049474913904$. The solid curve are for $\epsilon=0$ with $N=4.2910$, the dot-dashed curve for $\epsilon=0.5$ with $N=1.5$, and the dotted curve for $\epsilon=0.6448$ and $N=0.5$

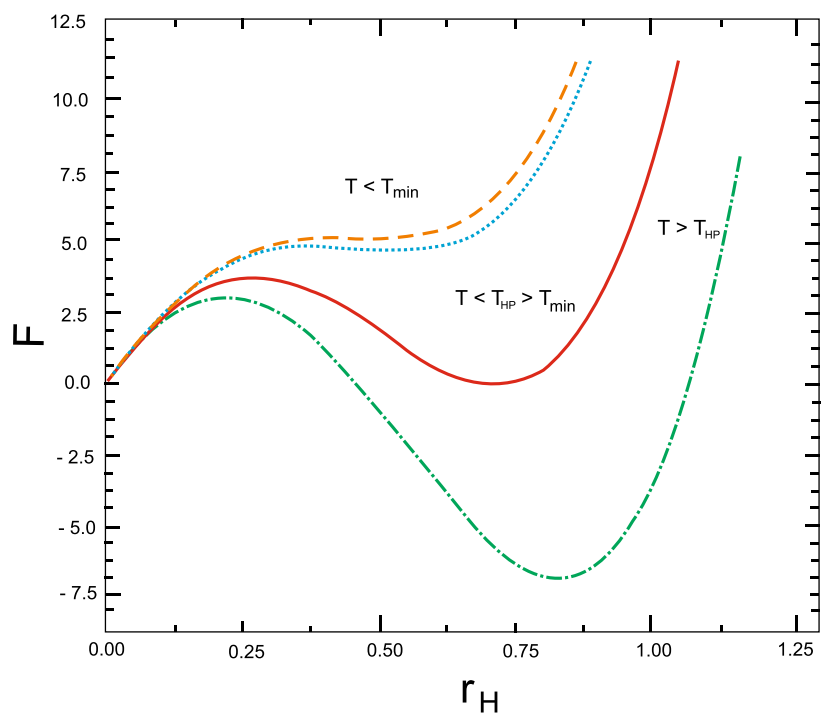

Fig. 5 Graphs of $F \times r_{H}$ for positive cosmological constant with $T_{H_{\min }}=0.00909823501, T_{H P}=0.049474913904, T_{S H}=$ 0.42359355918 considering $\epsilon=0.5$ and $N=1.5$

This transition can be studied by keeping the temperature constant for different values of $\epsilon$. With this methodology we obtained the curves of Fig. 4, for several values of $\epsilon$.

Performing the same procedure of the last section by calculating the roots of the Helmholtz free energy equation and solving numerically the problem of finding one of this energy that is unique and that touches the $r_{H}$ axis, we construct the graph of the Fig. 5.

In Fig. 6 it can be seen that in the absence of the global monopole, we obtain that the specific heat $C>0$ and the temperature $T>0$, and therefore, exists a stable black hole
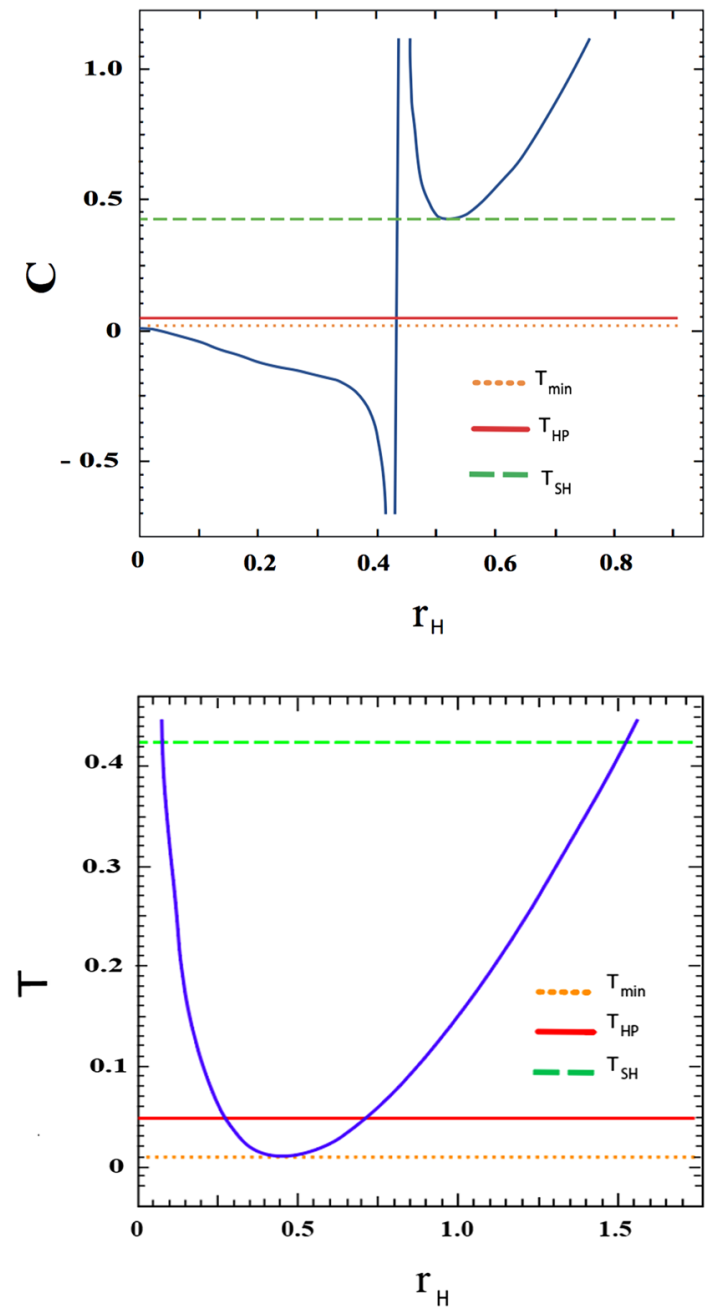

Fig. 6 Graphs of $C \times r_{H}$ (up panel) and of $T \times r_{H}$ (down panel) for positive cosmological constant, with $T_{\min }=0.00910104338, T_{H P}=$ 0.049474913904 and $T_{S H}=0.42359355918$. The curve gives us $\epsilon=$ 0.5 and $N=1.5$

This behavior is consistent with the case when the energy increases more rapidly than the temperature, so that the specific heat increases. In this situation, a new phase transition appears, even in the case of the temperature $T>T_{\min }$, at $r_{H}=r_{H_{c}}$. For $r_{H_{\min }}<r_{H} \leq r_{H_{c}}$, the specific heat decreases with $r_{H}$, as the temperature increases.

These results are similar in both cases, namely, with positive or negative cosmological constant. These are no asymptotically neither dS nor AdS, due to the presence of the logarithm term.or equivalently, due to the presence of the torsion.

\subsection{The AdS limit for black holes}

In order to better understand the phase transitions in black holes systems, we initially worked with the case where we have a locally AdS geometry. We will study now, besides the phase transitions, the role of the global monopole. We can 
easily see in metric (31) that the existence of the torsion can break the AdS geometry when $\beta \neq 3$, due to the presence of the logarithm. For this reason, to study the locally AdS case with torsion, we will initially consider $\beta=3$. In this case $N=0$ in the metric (31).

The dependence of the energy (GM) in function of the horizon radius $r_{H}$, is

$G M=\frac{1}{2} r_{H}\left[1-\epsilon+\frac{r_{H}^{2}}{L^{2}}\right]$.

The Hawking temperature is, therefore, the derivative of the energy with respect to the entropy of the black hole. From the first law of thermodynamics we have that the Hawking temperature is given by $d G M=T d S$ and then we find

$T=\frac{1}{4 \pi r_{H}}\left[1-\epsilon+\frac{3 r_{H}^{2}}{L^{2}}\right]$.

We will now examine the heat capacity, which can be calculated from the energy by using Eq. (37), resulting the following expression

$C=2 \pi r_{H}^{2}\left[\frac{1-\epsilon+\frac{3}{L^{2}} r_{H}^{2}}{\epsilon-1+\frac{3}{L^{2}} r_{H}^{2}}\right]$.

Now let us consider the energy of the Hawking-Page phase transition that occurs when the Helmholtz free energy is zero. Giving that this e-energy is

$$
\begin{aligned}
F & =G M-T S \\
& =\frac{1}{2} r_{H}\left(1-\epsilon+\frac{1}{L^{2}} r_{H}^{2}\right)-\pi T r_{H}^{2},
\end{aligned}
$$

thus, the Hawking-Page temperature and the horizon radius are expressed as

$T_{H P}=\frac{1}{L \pi} \sqrt{1-\epsilon}$,

$r_{H P}=\sqrt{1-\epsilon} L$.

If we use the Eq. (34), we find

$\frac{3}{L^{2}} r_{+}^{2}-4 \pi T r_{+}+1-\epsilon=0$.

This result gives us a pair of BH's (large/small) with the radii given by

$r_{\text {Large } / \text { Small }}=\frac{2}{3} \pi T L \pm \frac{1}{3} \sqrt{4 L^{2} \pi^{2}+3 \epsilon-3} L$.

The corresponding Hawking temperature $T_{H}$ is given by the derivative of Hawking temperature as a function of $r_{H}$. Their minimum values are thus obtained from
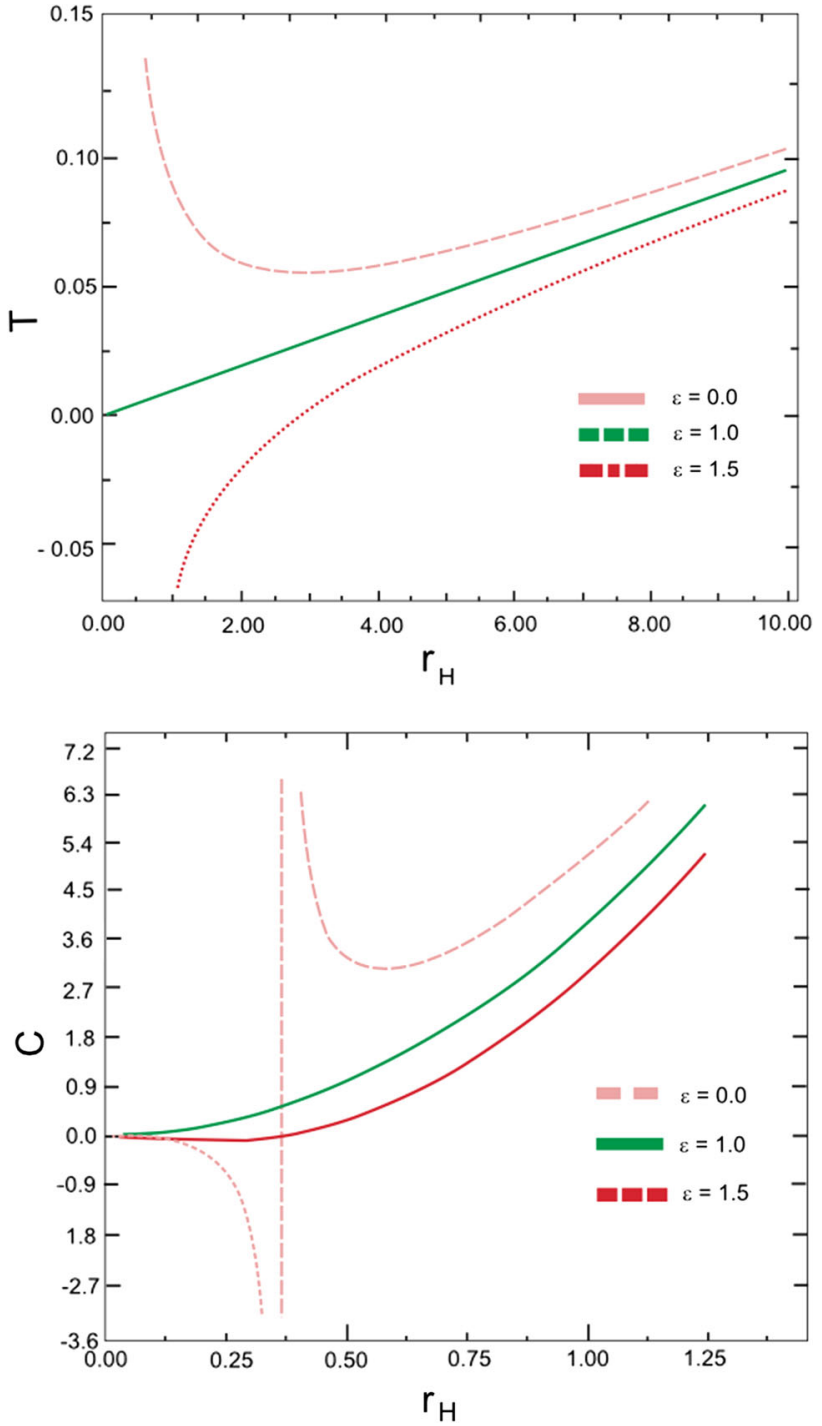

Fig. 7 Graphs of the temperature $T \times r_{H}$ upper panel and the specific heat capacity (lower panel) considering the $A d S_{4}$ case. In the upper for $N=0$ and $\mathrm{L}=5$

$r_{\text {min }}=\frac{\sqrt{1-\epsilon}}{\sqrt{3}} L$,

$T_{\min }=\sqrt{3} \frac{1-\epsilon}{2 \pi \sqrt{1-\epsilon} L}$.

In Fig. 7 we analyze all the important points in the graphs of the temperature as a function of the horizon radius.

In general, in the case of a negative cosmological constant, a black hole in an asymptotically flat space has a spherical event horizon. If the cosmological constant is negative, the black hole has no longer a spherical horizon. This type of black hole is called a topological black hole.

In Fig. 7, we show the temperature as a function of the horizon (upper panel). For a temperature $T<T_{\min }$ there are no black holes. It is a pass of pure radiation. For $T>T_{\min }$ there are two black holes with $r_{H}$ given by Eq. (46). 
Note that, we have $T>T_{\min }$ when $r_{H}<r_{H \text { min }}$. Thus, the temperature is always positive, as we can see in lower panel of Fig. 1, The heat capacity is negative, $C<0$, and we have an unstable black hole that we call small black hole. In the case where $r_{H}>r_{H \text { min }}$ the temperature is positive and according to Fig. 4 (lower panel) corresponds to the positive heat capacity, $C>0$. In this case we have a stable black hole that we call big black hole.

The importance of working with a topological defect in the AdS geometry is that the existence of a topological defect in the AdS bulk is an amount conserved at the boundary. Although the defect remains at the border, this does not break the CFT locally, because only the deficit solid angle is felt when we have a velocity relative to the defect [27]. This fact can bring us many applications of this metric to systems of condensed matter, where the vortex has an important role for the determination of the properties of the system and the fact that the vortex introduces a mass gap, responsible for the mass of the fermionic fields. As in the case of the AdS frontier being a conformal field theory, the presence of vortices can provide a mechanism to generate mass.

\section{The spin current}

The discovery of new materials such as graphene and topological insulators present to us new behaviors emerged in relation to the role played by the spin, dynamizing the area called spintronics. The theory developed in this paper permit us to get conclusions about how is the spin current behavior induced by torsion and curvature. The spin current tensor is given by

$S_{i j}^{\mu}=\frac{1}{\sqrt{-g}} \frac{\delta S_{M}}{\delta \Gamma_{\mu}{ }^{i j}}$.

Thus, considering the part of the quadratic terms of the curvature and torsion, the spin current can be written as

$\kappa S_{i j}^{\mu}=-\left[\bar{T}_{i j}^{\mu}+\alpha R_{i j \| \nu}^{\mu \nu}\right]$,

where $\bar{T}_{i j}^{\mu}$ and $R_{i j \| v}^{\mu \nu}$ are given by

$$
\begin{aligned}
\bar{T}_{i j}^{\mu}= & K^{\mu}{ }_{i j}-\beta \mathcal{Q}^{\mu}{ }_{i j} \\
= & (1-\beta) \mathcal{Q}_{i j}^{\mu}-\mathcal{Q}_{m i}^{m} \delta_{j}^{\mu}-\mathcal{Q}^{m}{ }_{j m} \delta_{i}^{\mu}, \\
R_{i j \| v}^{\mu \nu}= & R_{i j ; v}^{\mu \nu}-\Gamma^{k}{ }_{\nu i} R_{k j}^{\mu \nu}-\Gamma^{k}{ }_{\nu j} R^{\mu \nu}{ }_{i k} \\
& -\left\{\begin{array}{c}
\lambda \\
\lambda \sigma
\end{array}\right\} R_{i j}^{\mu \sigma}-\left\{\begin{array}{c}
\mu \\
\sigma \nu
\end{array}\right\} R_{i j}^{\mu \sigma} .
\end{aligned}
$$

By performing these calculations we can find that the components of the spin current density are $S_{01}^{0}, S^{1}{ }_{01}, S_{02}^{2}=S_{03}^{3}$ and $S_{12}^{2}=S^{3}{ }_{13}$. The contribution of the torsion to the spin current is given by the following components of $\bar{T}_{i j}^{\mu}$

$$
\begin{aligned}
\bar{T}_{01}^{0} & =\bar{T}_{01}^{1}=(\beta-2) h-2 k, \\
\bar{T}_{12}^{2} & =\bar{T}_{13}^{3}=-\bar{T}_{02}^{2}, \\
& =-\bar{T}_{03}^{3}=(3-\beta) k+h .
\end{aligned}
$$

We will focus on the case $\beta=3$, where we have: $\pm \frac{1}{L^{2}}=$ $\frac{1}{2 \alpha}$, with $\alpha$ being positive or negative. In the negative case we have de-Sitter space with cosmological constant $\Lambda=$ $\frac{3}{2|\alpha|}$ and in the positive case the Anti-de-Sitter, with negative cosmological constant $\Lambda=-\frac{3}{2|\alpha|}$, where $a=\left(1-\kappa \eta^{2}-\right.$ $\left.\frac{2 G M}{r}+\frac{r^{2}}{L^{2}}\right)^{1 / 2}$ and the quantities $k$ and $h$ are given by

$k=\frac{1}{3}\left(\frac{G M}{r^{2}}+\frac{r}{2 \alpha} \ln (r)\right) a$,

$h=\frac{1}{3}\left(\frac{G M}{r^{2}}+\frac{r}{2 \alpha}+\frac{r \ln r}{\alpha}\right) a$.

In this way, using Eqs. (49) and (52) for $\beta=3$, we obtain the graphs of Fig. 8 with $s_{1 t}(r)=\kappa\left(S_{01}^{0}\right)_{t}=-\kappa\left(S_{01}^{1}\right)_{t}=$ $-h+2 k, s_{2 t}(r)=\kappa\left(S_{12}^{2}\right)_{t}=\kappa\left(S_{03}^{3}\right)_{t}=-h, s_{3 t}(r)=$ $\kappa\left(S_{02}^{2}\right)_{t}=\kappa\left(S_{03}^{3}\right)_{t}=h$.

In Fig. 8 the behavior of the components of the spin current density can be analyzed taken into account the torsion. This figure shows that the spin current falls to zero when $r \rightarrow \infty$ and as the test particles approach the black hole, the spin current density increases. The contribution of the monopole can act as a fine regulator of the spin current, which has the role of attenuating its density. The twist contributes weakly to the spin current if we compare it with the contribution of the curvature, as can be seen in Fig. 9. Note that close to the BH this contribution may be relevant. The importance of calculating in the Anti-Sitter case is that it may be relevant to understanding the quantum nature of gravitation at the boundary of that space via the correspondence $A d S_{4} / C F T_{3}$. The contributions coming from the curvature are given by the expression (51), or more explicitly,

$$
\begin{aligned}
\kappa S_{01}^{1}= & -\alpha\left[R_{1}^{\prime}-\left(a^{\prime}-h\right) R_{1}+2 k R_{2}\left(\frac{a}{r}-k\right) R_{3}\right. \\
& \left.-2\left(2 a^{\prime}+2 \frac{a}{r}-3 \frac{R_{1}}{r}\right)\right], \\
\kappa S^{2}{ }_{12}= & \kappa S^{3}{ }_{03}=-\alpha\left[2 k R_{5}+2\left(\frac{a}{r}-k\right) R_{4}\right. \\
& \left.\left.+h R_{1}\right)\right], \\
\kappa S_{02}^{2}= & \kappa S_{03}^{3}=-\alpha\left[R_{5}^{\prime}-\left(a^{\prime}+2 \frac{a}{r}-h\right) R_{3}\right. \\
& -h R_{2}-\left(\frac{a}{r}-k\right)\left(R_{6}+R_{4}\right)-k R_{2} \\
& \left.-\left(2 a^{\prime}+2 \frac{a}{r}-\frac{3}{r}\right) R_{5}\right],
\end{aligned}
$$



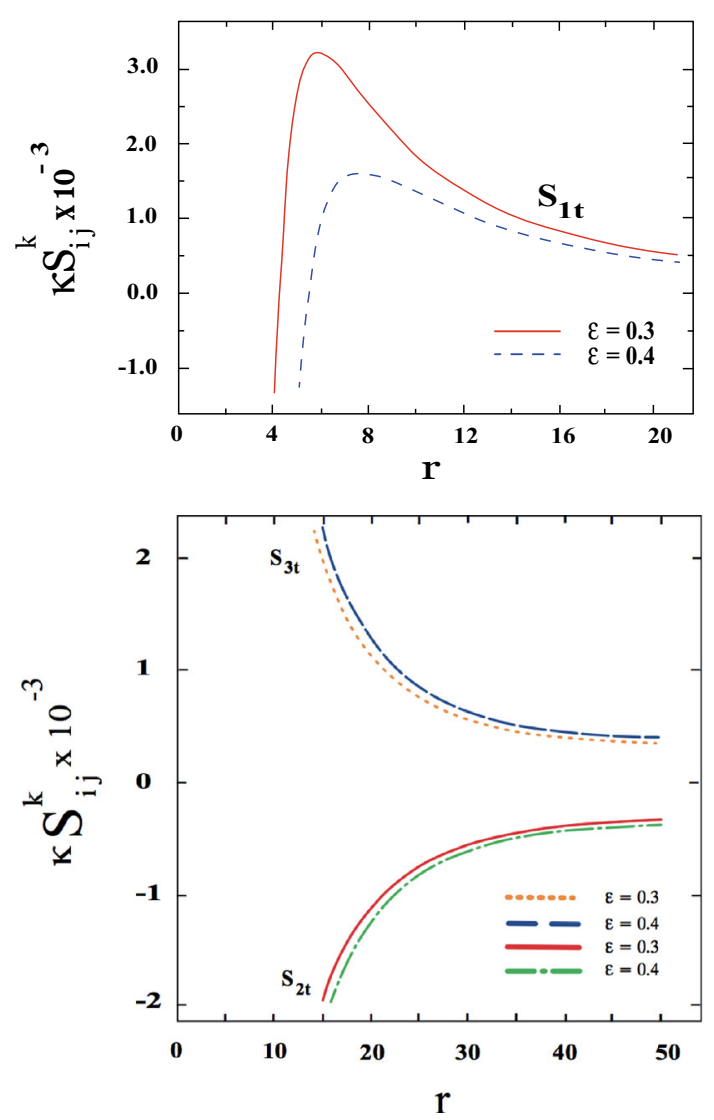

Fig. 8 These Graphs show the contribution of the torsion to the components of the spin current density on the upper panel, $s_{1 t}$ is shown. The lower panel, shows $s_{2 t}$ and $s_{3 t}$, with $\kappa \eta^{2}=0.3$ e 0.4 and $L=10^{3}$

$$
\begin{aligned}
\kappa S_{12}^{2}= & \kappa S_{13}^{3}=-\alpha\left[R_{4}^{\prime}+\left(a^{\prime}-h\right) R_{4}+h R_{5}\right. \\
& -k R_{6}+\left(\frac{a}{r}-k\right) R_{4}+k R_{3} \\
& \left.+\left(2 a^{\prime}+2 \frac{a}{r}-\frac{3}{r}\right) R_{4}\right]
\end{aligned}
$$

with $R_{1}=a a^{\prime} h-a h^{\prime}, R_{2}=-a k^{\prime}-a k / r+a h / r-h k$, $R_{3}=-\left(-h+a^{\prime}\right)(a / r-k), R_{4}=-\left(a^{\prime}+h\right) k, R_{5}=$ $a^{\prime} / r\left(k+r k^{\prime}-a^{\prime}\right)+h k$ and $R_{6}=1 / r^{2}+k^{2}(1 / r a-k)^{2}$.

It can be seen in Fig. 9 that the contribution to the spin current due to the curvature is greater if we consider a smaller negative cosmological constant. This result is compatible with what we find today in the universe, but in the primordial universe at high densities, this contribution may be of the order of the components arising from the twist. The contribution of the defect in this case can change its behavior, attenuating the current in certain regions and amplifying in others.

The obtained components of the spin current density without torsion is proportional to the inverse of the powers of the radius. In this way, when $r \rightarrow \infty$, that is, for regions far from the black hole, the spin current is too small to be observed.
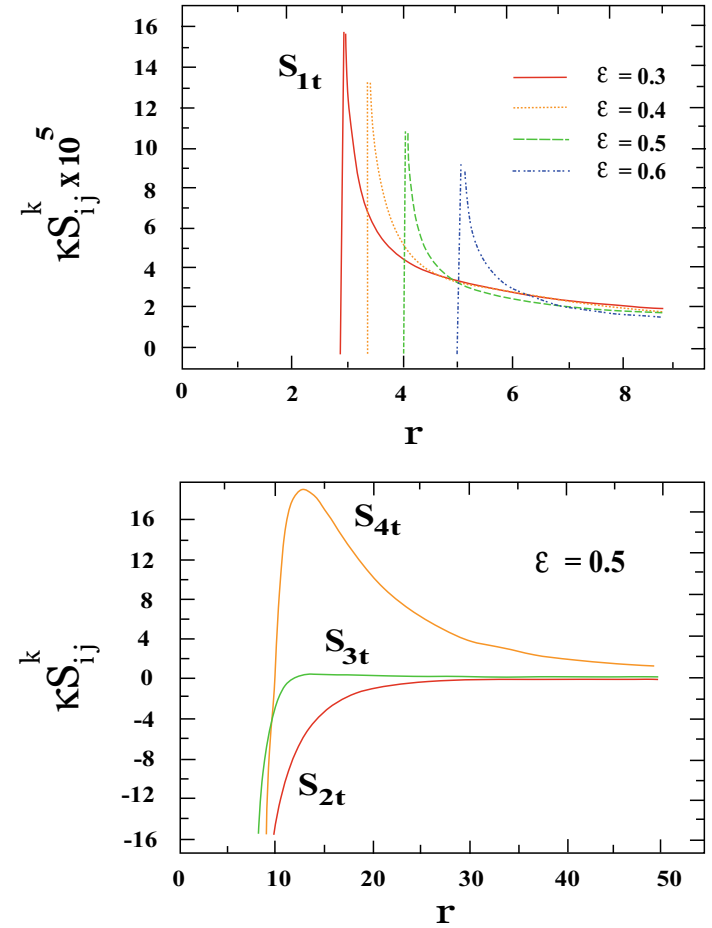

Fig. 9 The AdS contribution of the curvature to the components of the spin current one shown. In the upper panel $\kappa \eta^{2}=0.3 \ldots 0.6$ for the component $s_{1 c}(r)$, and in the lower panel the components $s_{2 c}(r), s_{3 c}(r)$ and $s_{4 c}(r)$ are shown for the same values of $\kappa \eta^{2}$

The study of the spin chains in an AdS gravitation together with the holographic principle is important to understand this quantity in new materials that are governed by the Dirac equation as topological insulators and graphene.

\section{Conclusions}

In this work we obtained the solution of a system corresponding to a black hole with a global monopole in the framework of the Poincaré gravity gauge theory. The topological defect induces the existence of a deficit solid angle that is responsible for several interesting effects, and these could be investigated in the future. The resulting metric presents a logarithmic correction which is important in the vicinity of the system.

Both terms, $r^{2}$ and $r^{2} \ln (r)$, can be interpreted as confinement terms mediated by the connection coefficients. The confinement potential may play an important role for strong gravitational fields and also in the strong interaction regime.

The thermodynamics behavior was studied considering different cases. Firstly, the Hawking temperature and the specific heat were studied in the general case containing logarithmic corrections in the metric. Although in this case we do not have a de-Sitter or Anti-de-Sitter geometry, these corrections cause the stability of the black hole in the case where the cosmological constant is positive. This stability was stud- 
ied using the comparison between specific heat and Hawking temperature and the Hawking-Page phase transition was also analyzed. In this analysis some critical points were found. One of those is the minimum temperature, $T_{\min }$, that separates the regions were the black hole is stable from that were it is unstable.

Both phases have temperature and heat capacity positives. For $r_{H}<r_{\text {min }}$, there is a region where the temperature drops rapidly to $T_{\min }$. This we have a small black hole. Otherwise when $r_{H}>r_{\min }$ we have a big black hole. There are another phase transition, to heat capacity, when for $r>r_{\min }$ that passes for two regimes, in one decrease while the temperature increase and in the other the heat capacity increase while the temperature increase. The behavior of the spin current density in the presence of strong gravitational fields was also studied. Due to the asymptotic behavior, these currents were studied in the AdS and dS spacetimes which are conceptualized with the holographic formalism.

It was found the coupling constant of the square curvature represented by the Riemann tensor $R_{\mu \nu}^{a b}$ with the cosmological constant. This shows that for small values of the cosmological constant there is a contribution of spin current density due to the curvature, which is more relevant than the contribution arising from the twist, in the neighborhood of the black hole. In spite of this behavior if we consider that the cosmological constant may have a different value in the primordial universe, we have that both the contribution from the torsion and from the curvature are of the same order of magnitude. Although spin content is negligible at low energies, in the vicinity of high gravitational fields where the spin current density is relevant or in the primordial universe, when spin current density, certainly, played a very important role, such spin content may be crucial for understanding the quantum properties of these systems. In this sense, the theory used, which extends the geometrical scheme to inclusion the intrinsic angular momentum, is very interesting because it unifies spin and gravitation through the antisymmetric connection that is related to the torsion, defined within the framework of a Riemann-Cartan manifold.

In a near future, we plan to study the holographic content of high spin densities due to its importance to understand the quantum nature of systems subject to strong gravitational fields.

Acknowledgements VBB thanks the CNPq for a partial financial support and CNF thanks the Universidade Federal da Paraíba for the financial support and hospitality during the preparation of this work.

Open Access This article is distributed under the terms of the Creative Commons Attribution 4.0 International License (http://creativecomm ons.org/licenses/by/4.0/), which permits unrestricted use, distribution, and reproduction in any medium, provided you give appropriate credit to the original author(s) and the source, provide a link to the Creative Commons license, and indicate if changes were made.

Funded by SCOAP ${ }^{3}$.

\section{References}

1. N. Haba, H. Ishida, R. Takahashi, Y. Yamaguchi, JHEP 1602, 058 (2016). https://doi.org/10.1007/JHEP02(2016)058. arXiv:1511.02107 [hep-ph]

2. P. Cox, A. Kusenko, O. Sumensari, T.T. Yanagida, JHEP 1703, 035 (2017). https://doi.org/10.1007/JHEP03(2017)035. arXiv:1612.03923 [hep-ph]

3. W. Hu, I. Sawicki, Phys. Rev. D 76, 064004 (2007). arXiv:0705.1158 [astro-ph]

4. A.D. Linde, Rep. Prog. Phys. 47, 925 (1984). https://doi.org/10. 1088/0034-4885/47/8/002

5. D. Wang, X.H. Meng, Phys. Rev. D 95, 023508 (2017). https://doi. org/10.1103/PhysRevD.95.023508

6. J. Dutta, W. Khyllep, N. Tamanini, Phys. Rev. D 95, 023515 (2017). https://doi.org/10.1103/PhysRevD.95.023515. arXiv:1701.00744 [gr-qc]

7. F.W. Hehl, P. von der Heyde, Rev. Mod. Phys. 48, 393 (1976). https://doi.org/10.1007/JHEP03(2017)035. arXiv:1612.03923 [hep-ph]

8. S. Akhshabi, E. Qorani, F. Khajenabi, EPL 119, 29002 (2017). https://doi.org/10.1209/0295-5075/119/29002. arXiv:1705.04931 [gr-qc]

9. J.D. Bekenstein, Phys. Rev. D 7, 949 (1973)

10. J.D. Bekenstein, Lett. Nuovo Cim. 4, 737 (1972)

11. J.D. Bekenstein, Phys. Rev. D 7, 2333 (1973)

12. J.D. Bekenstein, Phys. Rev. D 9, 3292 (1974)

13. S.W. Hawking, Commun. Math. Phys. 43, 199 (1975) [Erratumibid. 46, 206 (1976)]

14. R.-G. Cai, L.-M. Cao, N. Ohta, JHEP 1004, 082 (2010). arXiv:0911.4379 [hep-th]

15. Y.S. Myung, Y.-W. Kim, Y.-J. Park, Phys. Rev. D 78, 084002 (2008). arXiv:0805.0187 [gr-qc]

16. R. Biswas, S. Chakraborty, Gen. Relativ. Gravit. 43, 41 (2011)

17. M. Barriola, A. Vilenkin, Phys. Rev. Lett. 63, 341 (1989)

18. H.H. Zhao, L.C. Zhang, F. Liu, R. Zhao, Thermodynamics and critical behaviors of topological dS black hole with nonlinear sources. arXiv:1704.05167 [hep-th]

19. C.A. Ballon Bayona, C.N. Ferreira, Phys. Rev. D 78, 026004 (2008). https://doi.org/10.1103/PhysRevD.78.026004. arXiv:0801.0305 [hep-th]

20. J.M. Maldacena, Adv. Theor. Math. Phys. 2, 231 (1998). arXiv:hep-th/9711200

21. J.M. Maldacena, Int. J. Theor. Phys. 38, 1113 (1999)

22. O. Aharony, S.S. Gubser, J.M. Maldacena, H. Ooguri, Y. Oz, Phys. Rep. 323, 183 (2000). arXiv:hep-th/9905111

23. S. Hawking, D.N. Page, Commun. Math. Phys. 87, 577 (1983)

24. P. Baekler, F.W. Hehl, Class. Quantum Gravity 28, 215017 (2011). $\quad$ https://doi.org/10.1088/0264-9381/28/21/215017. arXiv:1105.3504 [gr-qc]

25. P. Baekler, F.W. Hehl, J.M. Nester, Phys. Rev. D 83, 024001 (2011). https://doi.org/10.1103/PhysRevD.83.024001. arXiv: 1009.5112 [gr-qc]

26. K. Hashimoto, N. Iizuka, T. Kimura, Phys. Rev. D 91, 086003 (2015). https://doi.org/10.1103/PhysRevD.91.086003. arXiv: 1304.3126 [hep-th]

27. C.A.B. Bayona, C.N. Ferreira, V.J.V. Otoya, Class. Quantum Gravity 28, 015011 (2011). https://doi.org/10.1088/0264-9381/28/1/ 015011. arXiv:1003.5396 [hep-th] 